\title{
De novo ATP1A2 variants in two Chinese children with alternating hemiplegia of childhood upgraded the gene-disease relationship and variant classification: a case report
}

Danping Huang ${ }^{1 \dagger}$, Min Liu ${ }^{1 \dagger}$, Hongying Wang², Bingbing Zhang ${ }^{1}$, Dongjing Zhao ${ }^{1}$, Weihao Ling ${ }^{1}$, Manli Wang ${ }^{1}$, Jun Feng ${ }^{1}$, Yiping Shen ${ }^{3,4^{*}}$ and Xuqin Chen ${ }^{1 *}$

\begin{abstract}
Background: ATP1A2 gene mutation has been indicated to cause alternating hemiplegia of childhood (AHC); however, limited evidence supports this relationship so far.

Case presentation: We reported two Chinese patients with de novo ATP1A2 variants (c.970G>A and c.889G $>$ A). Both patients presented with episodes of alternating hemiplegia, seizures and mild developmental delay. Brain magnetic resonance imaging revealed abnormal signals in both patients.

Conclusions: The new genetic evidence we reported here strengthened the gene-disease relationship, and the gene curation level between ATP1A2 and AHC became "Moderate" following the ClinGen Standard Operation Procedure. Consequently, the two variants can be reclassified as likely pathogenic.
\end{abstract}

Keywords: Alternating hemiplegia of childhood, ATP1A2, De novo, Variant classification

\section{Background}

Alternating hemiplegia of childhood (AHC) is a rare syndrome that is characterized by recurrent and varying degrees of alternating hemiplegia [1], as well as other movement disorders such as dystonic posturing, choreoathetoid movements, and ocular motor abnormalities [2]. Progressive cognitive impairment is also an important

\footnotetext{
*Correspondence: yiping.shen@childrens.harvard.edu; xuqinlili@163.com ${ }^{\dagger}$ Danping Huang and Min Liu have contributed equally to this manuscript 1 Department of Neurology, Children's Hospital of Soochow University, No. 92 Zhongnan Street, Soochow 215000, Jiangsu, China

${ }^{3}$ Genetic and Metabolic Central Laboratory, Birth Defect Prevention Research Institute, Maternal and Child Health Hospital, Children's Hospital of Guangxi Zhuang Autonomous Region, No. 225 Xinyang Street, Nanning 530000, Guangxi, China

Full list of author information is available at the end of the article
}

feature of this disease [3]. Pathogenic variants in ATP1A2 are known to cause AHC type 1 (OMIM\# 104290), but only a very limited number of cases have been reported [4-6]; thus, the gene (ATP1A2)-disease (AHC) relationship is "Limited" according to the ClinGen gene curation protocol (https://clinicalgenome.org/docs/summary-ofupdates-to-the-clingen-gene-clinical-validity-curationsop-version-7/). Pathogenic variants in ATP1A2 are more commonly associated with familial hemiplegic migraine (FHM) [7]. Here, we report two Chinese boys with de novo mutations in ATP1A2 who presented with mild growth and developmental delays, seizures, alternating hemiplegia, and abnormal MRI signals. The reporting of these two cases can elevate the level of gene-disease association from "Limited" to "Moderate", thus further 
strengthening the causal relationship between ATP1A2 and AHC.

\section{Case presentation}

We identified two Chinese AHC patients at the Children's Hospital Affiliated with Soochow University from August 2016 to August 2020. The patients were molecularly diagnosed by exome sequencing, and the diagnosis was confirmed by Sanger sequencing. The study followed the principles of the Declaration of Helsinki and was approved by the Institutional Review Committee of Children's Hospital Affiliated with Soochow University. Protocol Written informed consent was obtained from the patients' parents.

Clinical chart review provided information regarding family history, case histories and neurological symptoms and onset. Magnetic resonance imaging (MRI) (3.0T) scans and electroencephalogram (EEG) were performed in both patients.

\section{Case 1}

Case 1 was a 4-year-old boy with febrile seizures, epileptic seizures, and recurrent hemiplegia. He had two episodes of acute hemiplegia within 1 year. Each episode was preceded by mild fever and generalized tonic-clonic seizures lasting less than 5 min followed by several days of hemiparesis. Delayed motor development was exhibited: he was able to hold his neck up at 3 months and to walk unassisted at 15 months. There was no family history of similar symptoms, migraine or seizures.

Brain diffusion-weighted magnetic resonance imaging (MRI-DWI) showed transient unilateral cortical restricted diffusion signals during a severe episode of hemiplegia (Fig. 1a). EEG showed diffuse slow waves throughout the waking and sleeping phases, especially in one hemisphere (corresponding to the abnormal MRI signal side) (Fig. 2a). His second hemiplegic episode was not accompanied by obvious abnormalities on MRI, but EEG changes (Fig. 2b) were found, leaving him with persistent aphasia for over a year.

\section{Case 2}

Case 2 was a 19-month-old boy from a nonconsanguineous couple who was admitted to our hospital because of fever and right hemiplegia after one febrile convulsion (generalized tonic-clonic seizures and right-sided clonic seizures lasting less than $5 \mathrm{~min}$ ). Neurological examination indicated right hemiparesis for 2 days but no other neurological signs. He had a similar episode 2 months before admission, which did not draw attention. Brain MRI showed mild DWI hyperintensity involving the left hemispheric cortex (Fig. 1b), while his EEG was normal. He was able to hold his neck up at 3 months, sit without support at 8 months, and walk unassisted at 15 months. He had no positive family history.

The clinical data of the two Chinese patients with ATP1A2 mutations are summarized in Table 1.

\section{Results}

Variants in ATP1A2 (NM_000702) were identified by whole-exome sequencing: c.970G $>A$ : p.Gly324Ser [Chr1(GRCh37):g.160097563G>A] in case 1 and c.889G $>$ A: p.Ala297Thr [Chr1(GRCh37):g.160097482G $>$ A] in case 2. These variants were confirmed by Sanger sequencing in the patients and their parents and were found to be de novo variants. These two variants are classified as "likely pathogenic" following ACMG guidelines [8]. The variant c.970G $>$ A: p.Gly324Ser of case 1 has not been reported in the literature. The variant c.889G>A: p.Ala297Thr of case 2 was reported [9] to be associated with dystonia but not hemiplegia. Both variants are located in the intracellular region of the $\mathrm{Na}+/ \mathrm{K}+-$ ATPase a2 subunit.

As these two variants have not been reported to be related with AHC in the literature, to facilitate the evaluation of the clinical significance of each variant, we searched the same variant submitted in ClinVar (http:// www.ncbi.nlm.nih.gov/clinvar/), which displays supporting data from each submission and determines whether the submitted clinical interpretations are conflicting or concordant [10]. Searching 'c.970G $>$ A, c.889G $>$ A' in ClinVar returns one and four submissions, respectively. All these submissions were classified as variants of uncertain significance (VUS) in ClinVar. (Table 2).

Following these results, case 1 was treated with adenosine disodium triphosphate tablets and oxcarbazepine for his seizures, with no further hemiplegic episodes since the last admission. At his last follow-up, the strength of his limbs had completely returned. Although he still has speech problems, he has made great progress in following his parents' instructions. The hemiparesis of case 2 is basically restored, with no further attacks so far.

\section{Discussion and conclusions}

We report the clinical and genetic data of two Chinese AHC patients with de novo ATP1A2 variants. Febrile convulsions, AHC, mild developmental delay and MRI abnormalities were present in both patients. One patient had epilepsy with persistent aphasia. Postictal aphasia may be a feature of Todd's paralysis. We describe two children with episodes of hemiplegia after generalized tonic-clonic seizures. However, these seizures stop spontaneously within $5 \mathrm{~min}$. Their alternating attacks and developmental delay support the diagnosis of AHC rather than Todd's paralysis. To our knowledge, although the association of ATP1A2 variants with epilepsy and 

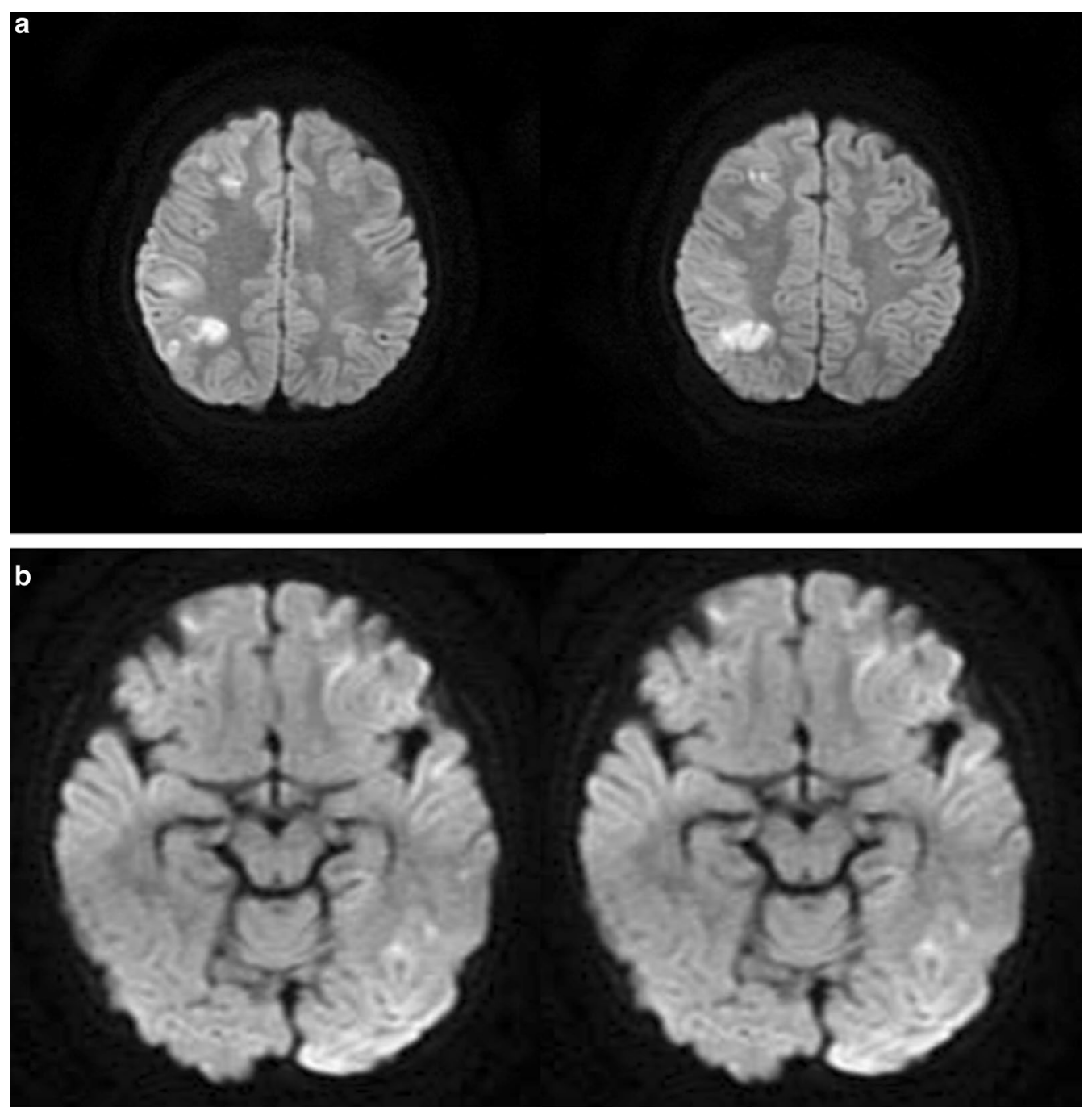

Fig. 1 Brain diffusion-weighted magnetic resonance imaging (MRI-DWI) in case 1 (a) and case 2 (b). a Brain MRI findings (axial; diffusion-weighted images) of case 1 (first hemiplegic episode). Cortical restricted diffusion signals were seen predominantly in the right frontal and parietal lobe during the episode of left hemiplegia. b Brain MRI findings (axial; diffusion-weighted images) of case 2. Mild hyperintensity was seen in the left hemispheric cortex during the episode of right hemiplegia

hemiplegic attacks has previously been described, phenotypic overlap with aphasia and MRI abnormalities has not yet been reported.

Our patients exhibited the clinical features of AHC. However, our patients are also distinguished from the majority of classic AHC patients by the late age of onset (typically $<18$ months), the lack of recurrent dystonia, and autonomic nervous manifestations associated with seizures [11-13]. Abnormal brain MRI is uncommon for AHC but has been reported previously [5]. However, persistent aphasia after hemiplegia has not been reported. Our study shows that the phenotype of patients with $A T P 1 A 2$ mutations is highly variable in terms of the diversity of clinical manifestations. Therefore, our patients' presentations extended the clinical phenotype of Chinese AHC associated with the ATP1A2 pathogenic variant.

ATP1A2 was initially identified to be associated with familial hemiplegia migraine (FHM) type 2 [14]. Several studies [4-6] have implicated ATP1A2 variants as uncommon causes of $\mathrm{AHC}$, and the $A T P 1 A 2$ variants are also associated with epilepsy and mental retardation [5]; however, few such cases have been reported, and none have been reported in Chinese patients so far. Based on the published cases, the gene-disease relationship regarding clinical validity is at a limited level per ClinGen 


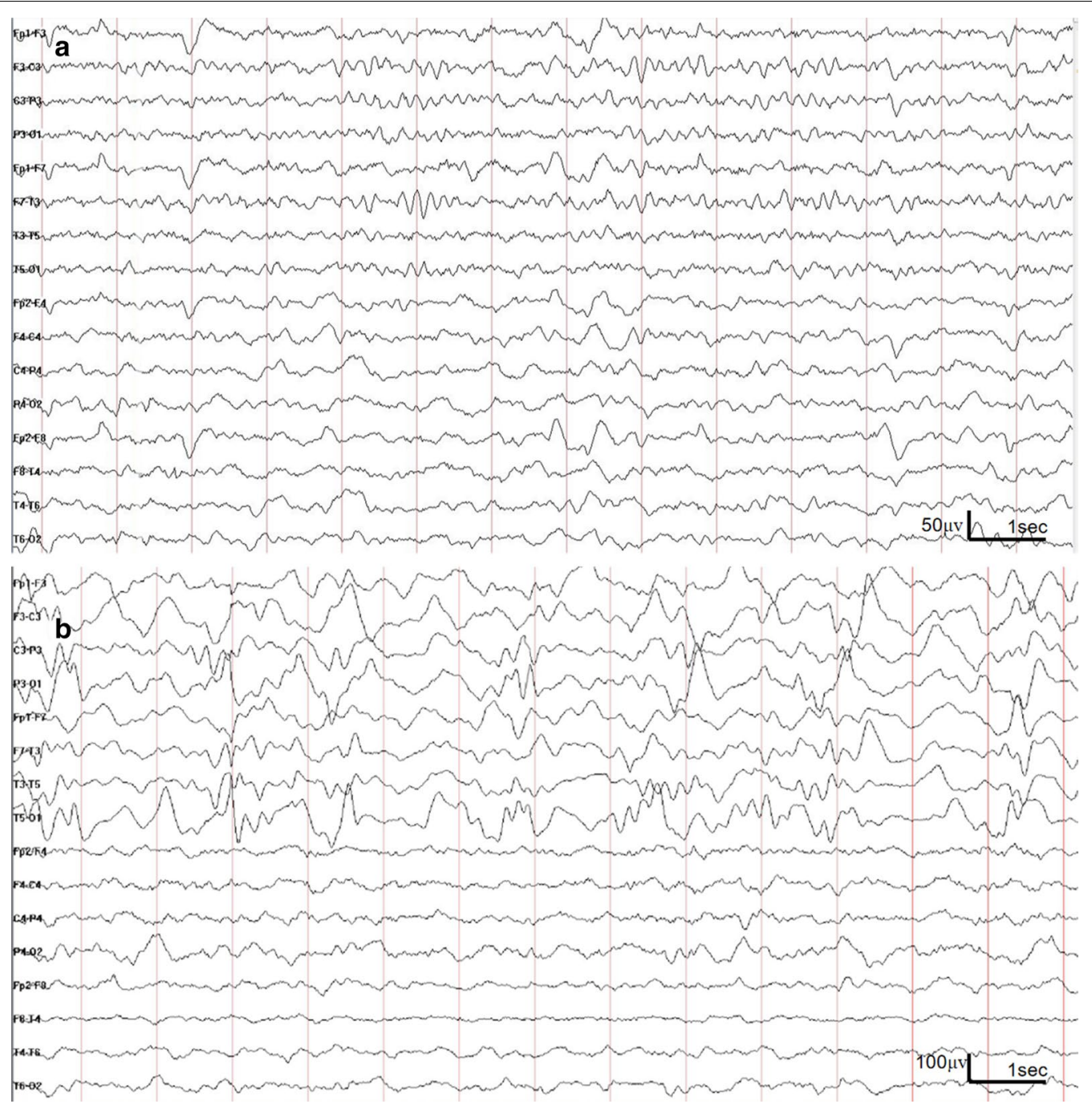

Fig. 2 Electroencephalogram (EEG) in case 1. a Diffuse slow waves were seen throughout the waking and sleeping phases, especially in the right hemisphere (first hemiplegic episode). b Diffuse high-amplitude slow waves were seen throughout the waking and sleeping phases in the left hemisphere (second hemiplegic episode)

gene curation classification (https://clinicalgenome. org/docs/summary-of-updates-to-the-clingen-geneclinical-validity-curation-sop-version-7/). To reach an affirmatory level, additional cases or functional evidence is needed. In our patients, two novel de novo variants (c.970G>A, c.889G >A) of ATP1A2 were identified and classified as "likely pathogenic" following ACMG guidelines [8]. These two cases provided sufficient evidence to reclassify the gene-disease relationship as "Moderate" (the total clinical validity score reached 8).

Furthermore, because these two variants have not been reported to be related with AHC in the literature, we searched ClinVar (http://www.ncbi.nlm.nih.gov/clinvar/) to further understand the ATP $1 A 2$ mutations mentioned above. The Variation Report presents all submissions referencing given variants. Interestingly, both of the novel variants have been submitted in ClinVar, while both of the submissions' variance classifications were VUS. According to the ACMG standard [8], all submissions meet criteria for PM1 (located in a mutational hot spot and/or critical and well-established functional domain without benign variation), PM2 (the variants are not present in population databases) and PP3 (algorithms developed to predict the effect of missense changes on protein structure and function suggest that the variants are likely to be disruptive, but these predictions have not been confirmed by published functional studies and their clinical significance is uncertain). In summary, as the available 
Table 1 Clinical data of the two patients with ATP1A2 mutations

\begin{tabular}{|c|c|c|}
\hline Clinical data & Patient1 & Patient2 \\
\hline Sex & Male & Male \\
\hline Age of onset & 4 year & 1 year 7 month \\
\hline Hemiparesis & Yes & Yes \\
\hline Oculomotor abnormalities (nystagmus, gaze deviation, strabismus) & No & Yes \\
\hline Disappearance of symptoms with sleep & Yes & Yes \\
\hline Febrile convulsion & Yes & Yes \\
\hline Developmental delays & Yes & Yes \\
\hline Barin MRI & Abnormal & Abnormal \\
\hline EEG & Abnormal & Normal \\
\hline Cerebrospinal fluid (CSF) & Normal & Normal \\
\hline Liquid chromatography-tandem mass spectrometry method (LC/MS) & Normal & Normal \\
\hline Genotype & $\begin{array}{l}\text { De novo missense variant } \\
\text { c.970G }>\text { A, } \\
\text { p.G324S }\end{array}$ & $\begin{array}{l}\text { De novo missense variant } \\
\text { c.889G }>\text { A, } \\
\text { p.A297T }\end{array}$ \\
\hline
\end{tabular}

Table 2 List of search results in ClinVar

\begin{tabular}{lllllll}
\hline Individual & CDNA & Protein & De novo & GnomAD & ACMG scoring & Pathogenicity \\
\hline Patient 1 & c.970G $>$ A & p.G324S & Yes & Not reported & PS2 + PM1+PM2 + PP3 & Likely path \\
1 & c.970G $>$ A & p.G324S & No & Not reported & PM1 + PM2 + PP3 & VUS \\
Patient 2 & c.889G $>$ A & p.A297T & Yes & Not reported & PS2 + PM1 + PM2 + PP3 & Likely path \\
1 & c.889G $>$ A & p.A297T & No & Not reported & PM1 + PM2 + PP3 & VUS \\
2 & c.889G $>$ A & p.A297T & No & Not reported & PM1 + PM2 + PP3 & VUS \\
3 & c.889G $>$ A & p.A297T & No & Not reported & PM1 + PM2 + PP3 & VUS \\
4 & c.889G $>$ A & p.A297T & No & Not reported & PM1 + PM2 + PP3 & VUS \\
\hline
\end{tabular}

de novo parents available to confirm de novo status, likely path likely pathogenic, VUS variants of uncertain significance. Each pathogenic criterion is weighted as very strong (PVS), strong (PS); moderate (PM), or supporting (PP)

evidence is currently insufficient to determine the pathogenicity of the two variants in disease, they have been classified as a VUS. Hence, the distinction between a deleterious mutation and a rare polymorphic variant may be difficult in isolated cases when the variant is unknown, except when de novo occurrence can be established [15]. Our patients provide crucial evidence that these two VUS could be upgraded as likely pathogenic. Ultimately, based on our two patients, five other patients carrying either of the two mutations submitted in ClinVar (http://www. ncbi.nlm.nih.gov/clinvar/) all around the world could be diagnosed genetically.

In conclusion, we reported two novel de novo ATP1A2 variants and additional phenotypes associated with AHC in two Chinese patients. This case series not only further strengthened the causal relationship between ATP1A2 and AHC but also extended the clinical phenotype of Chinese AHC associated with ATP1A2 pathogenic variants. More importantly, our study upgraded the variant classification of the two novel ATP1A2 variants (c.970G $>$ A, c.889G $>$ A). Our study is a good example of a rare case series that helps to interpret genetic tests in molecular diagnosis practice and upgrade the variant classification in ClinVar.

\section{Abbreviations \\ AHC: Alternating hemiplegia of childhood; VUS: Variants of uncertain signif- cance; FHM: Familial hemiplegic migraine.}

\section{Acknowledgements}

The authors wish to gratefully acknowledge the patients and their parents for allowing us to publish their case report.

\section{Authors' contributions}

DPH and ML conceived and designed the study, coordinated the research, and wrote the manuscript; HYW and MLW designed and commented on the manuscript draft; BBZ, DJZ, JF and WHL coordinated collection of DNA samples, undertook genetic counseling and analyzed those data; XQC and YPS reviewed and revised the manuscript. All authors read and approved the final manuscript.

\section{Funding}

The costs of the publication and molecular analyses of this research were supported by the Key special program funds of Jiangsu Province (BE2018661), Special project of diagnosis and treatment technology for key clinical diseases in Suzhou (LCZX201810) and the 15th batch of six talent peak training funds in 
Jiangsu Province (WSN-028). The funding bodies played no role in the design of the study and collection, analysis, and interpretation of data and in writing the manuscript.

\section{Availability of data and materials}

The datasets generated and/or analysed during the current study are available in the NCBI repository, [SAMN18376542; SAMN18376543; SAMN18376544; SAMN18376545; SAMN18449888; SAMN18449889]. These materials described in the manuscript, including all relevant raw data are available upon request.

\section{Declarations}

\section{Ethics approval and consent to participate}

This study was approved by the Ethics Committee of the Children's Hospital of Soochow University. Written informed consent was obtained from both of the parents of each of the patients to participate in our study.

\section{Consent for publication}

Written informed consent was obtained from both of the parents of each of the patients for publication of this case report and accompanying images. A copy of the written consent is available for review by the Editor-in-Chief of this journal.

\section{Competing interests}

The authors declare that they have no competing interests.

\section{Author details}

1 Department of Neurology, Children's Hospital of Soochow University, No. 92 Zhongnan Street, Soochow 215000, Jiangsu, China. ${ }^{2}$ Department of Laboratory, Children's Hospital of Soochow University, Soochow, Jiangsu, China. ${ }^{3}$ Genetic and Metabolic Central Laboratory, Birth Defect Prevention Research Institute, Maternal and Child Health Hospital, Children's Hospital of Guangxi Zhuang Autonomous Region, No. 225 Xinyang Street, Nanning 530000, Guangxi, China. ${ }^{4}$ Division of Genetics and Genomics, Boston Children's Hospital, Boston, MA, USA

Received: 19 November 2020 Accepted: 25 March 2021

Published online: 01 April 2021

\section{References}

1. Masoud M, Prange L, Wuchich J, Hunanyan A, Mikati MA. Diagnosis and treatment of alternating hemiplegia of childhood. Curr Treat Options Neurol. 2017;19(2):8.
2. Samanta D. Management of alternating hemiplegia of childhood: a review. Pediatr Neurol. 2020;103:12-20.

3. Au K, Pringsheim T. Alternating hemiplegia of childhood: clinical case and video description. Neurol Clin Pract. 2017;7(5):413-4.

4. Bassi MT, Bresolin N, Tonelli A, et al. A novel mutation in the ATP1A2 gene causes alternating hemiplegia of childhood. J Med Genet. 2004;41(8):621-8.

5. Swoboda KJ, Kanavakis E, Xaidara A, et al. Alternating hemiplegia of childhood or familial hemiplegic migraine? A novel ATP1A2 mutation. Ann Neurol. 2004;55(6):884-7.

6. Kors EE, Vanmolkot KR, Haan J, et al. Alternating hemiplegia of childhood: no mutations in the second familial hemiplegic migraine gene ATP1A2. Neuropediatrics. 2004;35(5):293-6.

7. Gritz SM, Radcliffe RA. Genetic effects of ATP1A2 in familial hemiplegic migraine type II and animal models. Hum Genom. 2013;7:8.

8. Richards S, Aziz N, Bale S, et al. Standards and quidelines for the interpretation of sequence variants: a joint consensus recommendation of the American College of Medical Genetics and Genomics and the Association for Molecular Pathology. Genet Med. 2015;17(5):405-24.

9. Graziola F, Garone G, Stregapede F, et al. Diagnostic yield of a targeted next-generation sequencing gene panel for pediatric-onset movement disorders: a 3-year cohort study. Front Genet. 2019;10:1026.

10. Harrison SM, Riggs ER, Maglott DR, et al. Using ClinVar as a resource to support variant interpretation. Curr Protoc Hum Genet. 2016;89:8.16.1-8.16.23.

11. Al-Bulushi B, Al-Hashem A, Tabarki B. A wide clinical phenotype spectrum in patients with ATP1A2 mutations. J Child Neurol. 2014;29(2):265-8.

12. Kornbluh AB, Chung MG. Teaching neuroimages: transient cytotoxic edema in a child with a novel ATP1A2 mutation. Neurology. 2020;95:e1441-2.

13. Gropman A, Uittenbogaard M, Brantner CA, Wang Y, Wong $L$, Chiaramello A. Molecular genetic and mitochondrial metabolic analyses confirm the suspected mitochondrial etiology in a pediatric patient with an atypical form of alternating hemiplegia of childhood. Mol Genet Metab Rep. 2020:24:100609.

14. Schwarz G, Anzalone N, Baldoli C, et al. Pediatric sporadic hemiplegic migraine (ATP1A2 gene): a case report and brief literature review. Neurol Sci. 2018;39(Suppl 1):69-71.

15. Riant F, Ducros A, Ploton C, Barbance C, Depienne C, Tournier-Lasserve E. De novo mutations in ATP1A2 and CACNA1A are frequent in early-onset sporadic hemiplegic migraine. Neurology. 2010;75(11):967-72.

\section{Publisher's Note}

Springer Nature remains neutral with regard to jurisdictional claims in published maps and institutional affiliations.
Ready to submit your research? Choose BMC and benefit from:

- fast, convenient online submission

- thorough peer review by experienced researchers in your field

- rapid publication on acceptance

- support for research data, including large and complex data types

- gold Open Access which fosters wider collaboration and increased citations

- maximum visibility for your research: over $100 \mathrm{M}$ website views per year

At BMC, research is always in progress.

Learn more biomedcentral.com/submissions 